\title{
Effect of poly-arginine R18 on neurocyte cell growth via autophagy in traumatic brain injury
}

\author{
HU BATULU ${ }^{1}$, GUO-JIA DU ${ }^{1}$, DA-ZHI LI ${ }^{2}$, DUISHANBAI SAILIKE ${ }^{1}$, \\ YU-HUA FAN $^{3}$ and DANGMURENJIAFU GENG ${ }^{1}$ \\ ${ }^{1}$ Department of Neurosurgery, The First Affiliated Hospital of Xinjiang Medical University, Urumqi, Xinjiang 830054; \\ ${ }^{2}$ Department of Neurosurgery, Traditional Chinese Medicine Hospital of Xinjiang Medical University, Urumqi, \\ Xinjiang 830000; ${ }^{3}$ Department of Nutrition Section, The People's Hospital of Xinjiang \\ Bortala Autonomous Prefecture of Monglia, Bortala, Xinjiang 833400, P.R. China
}

Received May 29, 2018; Accepted February 20, 2019

DOI: $10.3892 /$ etm.2019.7423

\begin{abstract}
The present study assessed the effects of poly-arginine R18 and its promotion of neurocyte cell growth via autophagy in traumatic brain injury (TBI), and aimed to determine the possible mechanism by which this occurs. Brain water content was measured to analyze the effects of poly-arginine R18 in TBI. MTT and lactate dehydrogenase activity assays were performed to measure N2A cell growth. Western blotting and immunofluorescence staining were also performed to determine the protein expression of Bcl-2 associated X, LC3, Beclin-1 and p62. The results demonstrated that poly-arginine R18 treatment reduced neurocyte apoptosis and promoted neurocyte cell growth via the activation of autophagy in a rat model of TBI. Furthermore, poly-arginine R18 treatment promoted neurocyte cell growth, reduced apoptosis, induced the protein expression of LC3 and Beclin-1, and suppressed p62 expression by promoting autophagy in vitro. In addition, the inhibition of autophagy attenuated the effects of poly-arginine R18 on cell growth in vitro. Collectively, the results demonstrate the effects of poly-arginine R18 on neurocyte cell growth via autophagy activation in a model of TBI, and poly-arginine R18 is therefore a potential therapeutic target in TBI.
\end{abstract}

\section{Introduction}

Traumatic brain injury (TBI) can be induced by injuries ranging from simple head impacts to penetrating wounds. TBI is a severe trauma in neurosurgery, which is associated with high disability and mortality rates (1). In the USA, TBI has resulted in $>5.3$ million disabilities, with $\sim 1.7$ million people

Correspondence to: Dr Dangmurenjiafu Geng, Department of Neurosurgery, The First Affiliated Hospital of Xinjiang Medical University, 137 Liyushan Road, Urumqi, Xinjiang 830054, P.R. China

E-mail: g989045181c@163.com

Key words: poly-arginine R18, traumatic brain injury, autophagy developing TBI per year (2). Of these, $80 \%$ of TBI cases are considered mild brain injuries, while moderate and severe TBI is the primary cause of death and disability (3). Severe TBI, combined with pathological changes, including cephaledema, cerebral ischemia and intracranial hypertension, may lead to cranial nerve injury and limb dysfunction (3).

It is well known that necrosis and apoptosis are two methods of cell Death (4). Necrosis is a passive and abnormal death process, which is induced by damage factors or other unfavorable environmental pressures (5). Apoptosis, an active and programmed process of cell death, is autonomously regulated by genes and functions to maintain homeostasis (5). A previous study indicated that autophagy serves as the third method of cell death (5). Autophagy is the process where intracellular proteins and organelles are degraded by the lysosome, which serves a vital role in the maintenance of homeostasis in eukaryotes (5). However, under pathological conditions, persistent and excessive autophagy activation results in cell death, which is called autophagic cell death (5). It is of great importance to assess the role of autophagy in brain injury as: i) an interaction exists between autophagy-induced cell death and apoptotic signaling (6); and ii) autophagy may activate certain apoptotic promoters in damaged nerve cells, thus initiating the cell apoptosis program (6).

A previous study has demonstrated that polypeptide rich arginine provides neuroprotection (7). Furthermore, it has been revealed that the poly-l-arginine R18 polypeptide and NA-1 also exhibit neuroprotective effects, with R18 being more protective than NA-1 (7). Cui et al (8) assessed the protective effects of R18, COG1410 and APP6-110 on neurons following TBI. It was revealed that R18 could reduce calcium influx and exhibit strong neuroprotection, while COG1410 and APP6-110 exhibited weak neuroprotection. However, the mechanism by which the R18 polypeptide exerts neuronal protective effects has not yet been fully elucidated. We examined the effect of poly-arginine R18 and its underlying mechanism in promoting neurocyte cell growth in TBI via autophagy.

\section{Materials and methods}

Animals and the controlled cortical impact (CCI) model. All experimental procedures in the current study were approved 
by the Animal Ethics Committee of First Affiliated Hospital of Xinjiang Medical University (Xinjiang, China). Adult male Sprague-Dawley rats $(n=42$; age, 8-10 weeks; weight, 200-230 g) were obtained from Animal experiment center of Xinjiang medical university (Xinjiang, China). Animals were maintained under a controlled temperature $\left(22-23^{\circ} \mathrm{C}\right)$ and humidity (55-60\%), with a $12 \mathrm{~h}$ light/dark cycle and free access to food and water. All rats were randomly assigned into the control, model or poly-arginine R18 groups (each, $n=6$ ). All rats in the model and poly-arginine R18 groups were anesthetized with $35 \mathrm{mg} / \mathrm{kg}$ pentobarbital and a craniotomy was performed at the bregma and lambda on the right frontoparietal cortex. In the R18 group, CCI was performed using a PinPoint ${ }^{\mathrm{TM}}$ Precision Cortical Impactor (Hatteras Instruments, Inc., Cary, NC, USA) perpendicular to the brain surface and all rats received a single injection of $300 \mathrm{nmol} / \mathrm{kg}$ poly-arginine R18 (synthesized by Sangon Biotech Co., Ltd., Shanghai, China) at $48 \mathrm{~h}$ following surgery, as previously described (9). Rats in the control group were anesthetized with $35 \mathrm{mg} / \mathrm{kg}$ pentobarbital and the same surgical procedure was performed without CCI.

Brain water content measurement. Following treatment with poly-arginine $\mathrm{R} 18$, rats ( $\mathrm{n}=3$ /group) were sacrificed, brain samples were collected and tissue was washed with PBS. The wet weight of brain tissue was recorded and samples were then dried at $68^{\circ} \mathrm{C}$ for $48 \mathrm{~h}$ to obtain the dry weight. Brain water content was calculated as follows: wet weight-dry weight/wet weight $\mathrm{x} 100 \%$.

Hematoxylin and Eosin $(H \& E)$ staining. Following treatment with poly-arginine $\mathrm{R} 18$, rats ( $\mathrm{n}=3$ /group) were sacrificed and the hippocampus tissue were fixed with $4 \%$ paraformaldehyde for $24 \mathrm{~h}$ at room temperature and embedded in paraffin. Paraffin-embedded tissue samples were cut into $10 \mu \mathrm{m}$-thick sections. Tissue samples were stained with hematoxylin and eosin (H\&E) for $5 \mathrm{~min}$ at room temperature and examined using a confocal microscope (magnification, x50; Olympus BX51; Olympus Corp., Tokyo, Japan).

Cell culture. The neuroblastoma neuro-2A (N2A) cell line was purchased from the Type Culture Collection of the Chinese Academy of Sciences (Shanghai, China) and cultured in Dulbecco's modified Eagle's medium (Gibco; Thermo Fisher Scientific, Inc., Waltham, MA, USA) supplemented with $10 \%$ heat-inactivated fetal bovine serum (FBS; Gibco; Thermo Fisher Scientific, Inc.), $100 \mathrm{U} / \mathrm{ml}$ penicillin and $100 \mu \mathrm{g} / \mathrm{ml}$ streptomycin in a humidified atmosphere of $5 \% \mathrm{CO}_{2}$ at $37^{\circ} \mathrm{C}$. $\mathrm{N} 2 \mathrm{~A}$ cells in the model group were then stimulated with 500 ng/ml lipopolysaccharide (LPS; Sigma-Aldrich; Merck KGaA, Darmstadt, Germany). In the R18 group, N2A cells were treated with $500 \mathrm{ng} / \mathrm{ml}$ LPS and $0.5 \mu \mathrm{M}$ poly-arginine R18 as previously described (9). In the 3-MA group, N2A cells were treated with an autophagy inhibitor 3-MA $(5 \mu \mathrm{M}$; MedChemExpress, Shanghai, China), $500 \mathrm{ng} / \mathrm{ml}$ LPS and $0.5 \mu \mathrm{M}$ R18.

MTT and lactate dehydrogenase $(\mathrm{LDH})$ activity. A total of $10 \mu \mathrm{l}$ MTT $(5 \mathrm{mg} / \mathrm{ml}$; cat. no. C0009; Beyotime Institute of Biotechnology, Haimen, China) was added to N2A cells for
$4 \mathrm{~h}$ at $37^{\circ} \mathrm{C}$. DMEM was subsequently removed and dimethyl sulfoxide was added to cells and incubated for $20 \mathrm{~min}$ at $37^{\circ} \mathrm{C}$. The optical density in each well was measured at a wavelength of $492 \mathrm{~nm}$ using a microplate reader (Molecular Devices, Sunnyvale, CA, USA). LDH activity was measured using an LDH Cytotoxicity Detection kit (cat. no. C0017; Beyotime Institute of Biotechnology), according to the manufacturer's protocol. The optical density was measured at a wavelength of $450 \mathrm{~nm}$ using a microplate reader to determine the LDH activity.

Caspase-3/8/9 activity. N2A cells were lysed using radioimmunoprecipitation assay (RIPA) buffer for 15-30 min at $4^{\circ} \mathrm{C}$. The supernatant was then centrifuged at 2,000 $\mathrm{x}$ g for $10 \mathrm{~min}$ at $4^{\circ} \mathrm{C}$ and protein content was measured using a bicinchoninic acid (BCA) assay. The activity of caspase-3/8/9 was assessed in total protein $(10 \mu \mathrm{g})$ using a caspase-3 (cat. no. C1115), caspase- 8 (cat. no. C1151) and caspase-9 (cat. no. C1158) activity kits (all Beyotime Institute of Biotechnology). Optical density values in each well were determined using an enzyme immunoassay analyzer at $405 \mathrm{~nm}$.

Western blot analysis. Tissue samples were homogenized or N2A cells were lysed using RIPA buffer for 15-30 min at $4^{\circ} \mathrm{C}$. The supernatant was then centrifuged at 2,000 $\mathrm{x} \mathrm{g}$ for $10 \mathrm{~min}$ at $4^{\circ} \mathrm{C}$ and the protein content was measured using a BCA assay. Total protein $(50 \mu \mathrm{g})$ was separated via SDS-PAGE on a $10 \%$ gel. Separated proteins were transferred onto polyvinylidene fluoride membranes and then blocked with 5\% non-fat milk in TBST for $1 \mathrm{~h}$ at $37^{\circ} \mathrm{C}$. Membranes were incubated overnight at $4^{\circ} \mathrm{C}$ with primary antibodies against Bcl-2 associated X (Bax; 1:1,000; cat. no. 5023), LC3 (1:1,000; cat. no. 4599), Beclin-1 (1:1,000; cat. no. 3495), p62 (1:1,000; cat. no. 23214) and GAPDH (1:1,000; cat. no. 5174; all Cell Signaling Technology, Inc., Danvers, MA, USA). Subsequently, membranes were washed with Tris-buffered saline containing $0.1 \%$ Tween ${ }^{\circledR} 20$ (TBST) and membranes were incubated with horseradish peroxidase-labeled goat anti-rabbit IgG secondary antibody (1:5,000; cat. no. 7074; Cell Signaling Technology, Inc.) for $1 \mathrm{~h}$ at room temperature. Samples were visualized using an enhanced chemiluminescence kit (GE Healthcare, Chicago, IL, USA) and exposed to Image Lab 3.0 software (Bio-Rad Laboratories, Inc., Hercules, CA, USA).

Immunofluorescence. N2A cells were washed with PBS and fixed using $4 \%$ paraformaldehyde for $20 \mathrm{~min}$ at room temperature. Following further washes with PBS, cells were stained with DAPI (Sigma-Aldrich; Merck KGaA) for 20 min in darkness at room temperature. Stained cells were observed using an inverted confocal laser scanning microscope (magnification, 50x; TCS SP5; Leica Microsystems GmbH, Wetzlar, Germany).

Statistical analysis. Data are expressed as the mean \pm standard deviation. All statistical analyses were performed using SPSS software (version 20.0; IBM Corp., Armonk, NY, USA). All data were analyzed using one-way analysis of variance followed by a Tukey's multiple comparison test. $\mathrm{P}<0.05$ were considered to indicate a statistically significant difference. 


\section{Results}

Poly-arginine $R 18$ promotes neurocyte cell growth in TBI rats. TBI rats were treated with poly-arginine R18 and the effect on neurocyte cell growth was assessed. As presented in Fig. 1A and $\mathrm{B}$, neurocyte cell apoptosis and brain water content was markedly increased in the TBI model group compared with the control group. Furthermore, the activity levels of caspase-3/8/9 were increased in the TBI model group compared with those in the control group (Fig. 1C-E). Treatment with poly-arginine R18 significantly reduced neurocyte cell apoptosis, brain water content and Bax protein expression whilst also inhibiting caspase-3/8/9 activity in the R18 group compared with the model group (Fig. 1). These results indicate that poly-arginine R18 promotes neurocyte cell growth in TBI rats.

Poly-arginine R18 induces neurocyte cell autophagy in TBI rats. The mechanism of poly-arginine R18 in neurocyte cell growth was assessed in TBI rats by analyzing changes in neurocyte cell autophagy. As presented in Fig. 2, LC3 and Beclin-1 protein expression was significantly reduced, whilst p62 and Bax expression was significantly increased in the TBI model group compared with the control group. Subsequent treatment with poly-arginine R18 significantly increased LC3 and Beclin-1 protein expression and reduced p62 and Bax expression in the R18 group compared with the TBI model group (Fig. 2). Thus, the results indicate that poly-arginine R18 induces neurocyte cell autophagy in TBI rats.

Poly-arginine R18 induces neurocyte cell autophagy in vitro. The autophagy of neurocyte cells was assessed following poly-arginine R18 treatment in an in vitro model of TBI. As presented in Fig. 3, the expression levels of LC3 and Beclin-1 was significantly reduced, whilst p62 and Bax expression was significantly increased in the in vitro TBI model group compared with the control group. Following treatment with poly-arginine R18, the expression levels of LC3 and Beclin-1 were significantly increased and p62 and Bax protein expression were significantly decreased in the R18 group compared with the in vitro TBI model group (Fig. 3A-E). Taken together, the results demonstrated that poly-arginine R18 induces neurocyte cell autophagy in vitro.

Poly-arginine R18 promotes neurocyte proliferation and reduces $L D H$ activity in vitro. Neurocyte cell proliferation was significantly reduced, whilst LDH activity was increased in the in vitro TBI model group compared with the control group (Fig. 4A and B). In addition, the activity levels of caspase-3/8/9 and cell nucleus apoptosis were significantly increased in the in vitro TBI model group compared with the control group (Fig. 4C-F). Following treatment with poly-arginine R18, neurocyte proliferation was significantly increased, whilst LDH and caspase-3/8/9 activity levels were significantly decreased in the R18 group compared with the in vitro TBI model group (Fig 4).

Autophagy inhibition attenuates the effects of poly-arginine $R 18$ on cell proliferation in vitro. An autophagy inhibitor (3-MA) was used to assess the mechanism of poly-arginine $\mathrm{R} 18$ on cell growth in an in vitro model of TBI. The expression levels of LC3 and Beclin-1 were significantly reduced, whilst p62 and Bax expression was significantly increased in the R18 group following treatment with 3-MA (3-MA group) compared with the R18 group (Fig. 5A-E). Furthermore, treatment with 3-MA attenuated the effects of poly-arginine R18 on neurocyte proliferation, LDH activity, caspase-3/8/9 activity levels and cell nucleus apoptosis compared with the R18 group (Fig. 5F-K).

\section{Discussion}

Brain injury is a common trauma that has a growing incidence rate in China (10). Brain injury can induce neurological damage through two mechanisms, including primary injury and secondary injury (10). Secondary brain injury refers to a series of pathophysiological changes that occur following acute injury (10). This comprises local microvascular injury, vascular rupture hemorrhage, spasm or thrombosis (11). Injuries such as these subsequently induce tissue ischemia, hypoxia, the inflammatory response and tissue edema, thus activating intracellular zymogen (11). Zymogen destroys various membrane structures within the tissue and eventually leads to apoptosis and necrosis (12). Therefore, it is important to understand the changes and repair mechanisms that occur following brain injury and to effectively suppress cell death, which may protect neurons and promote the repair and reconstruction of neurological functional injury (13). In the current study, it was revealed that poly-arginine R18 promotes neurocyte proliferation in TBI rats. Chiu et al (14) demonstrated that poly-arginine R18 reduces axonal injury following TBI (14). However, the current study only performed H\&E staining to detect brain injury, which is insufficient for a full assessment. Therefore, future studies should perform scanning tunneling microscopy to investigate microstructural organelle damage following injury.

Autophagy is an organelle and protein cycling process that is controlled by the lysosome degradation pathway (6). The primary feature of autophagy activation is the excessive accumulation of autophagosomes encapsulated by a double-membraned structure or by autophagic vacuoles (8). It has been demonstrated that cell autophagy is associated with aging, immunity, cell death and differentiation (15). Autophagy has also been associated with the pathogenesis of several types of neurodegenerative disease and malignant tumors (8). Autophagy is an early self-adaptive mechanism that eliminates damaged organelles and protein, and provides nutrients and energy via the lysosome degradation pathway to restore tissue homeostasis (8). Excessively activated or impaired autophagy leads to autophagic cell death, which accelerates disease development and deterioration (15). A previous study has indicated that neurological damage is closely associated with cell autophagy (15), such that the autophagic marker protein, LC3, is markedly upregulated in brain tissue subjected to traumatic injury (15). The present study determined that poly-arginine R18 treatment induced neurocyte cell autophagy in TBI rats. The N2A neuroblastoma cell line of mouse origin was utilized in the present study. However, neuroblastoma cells are different from neurocytes, which may affect the results of the present study. Future studies should therefore utilize different cell lines for the construction of an in vitro model. 


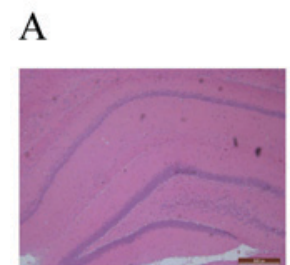

Control

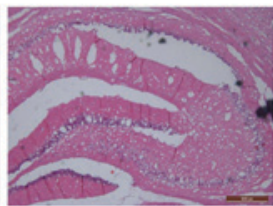

Model

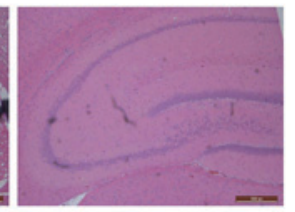

R18
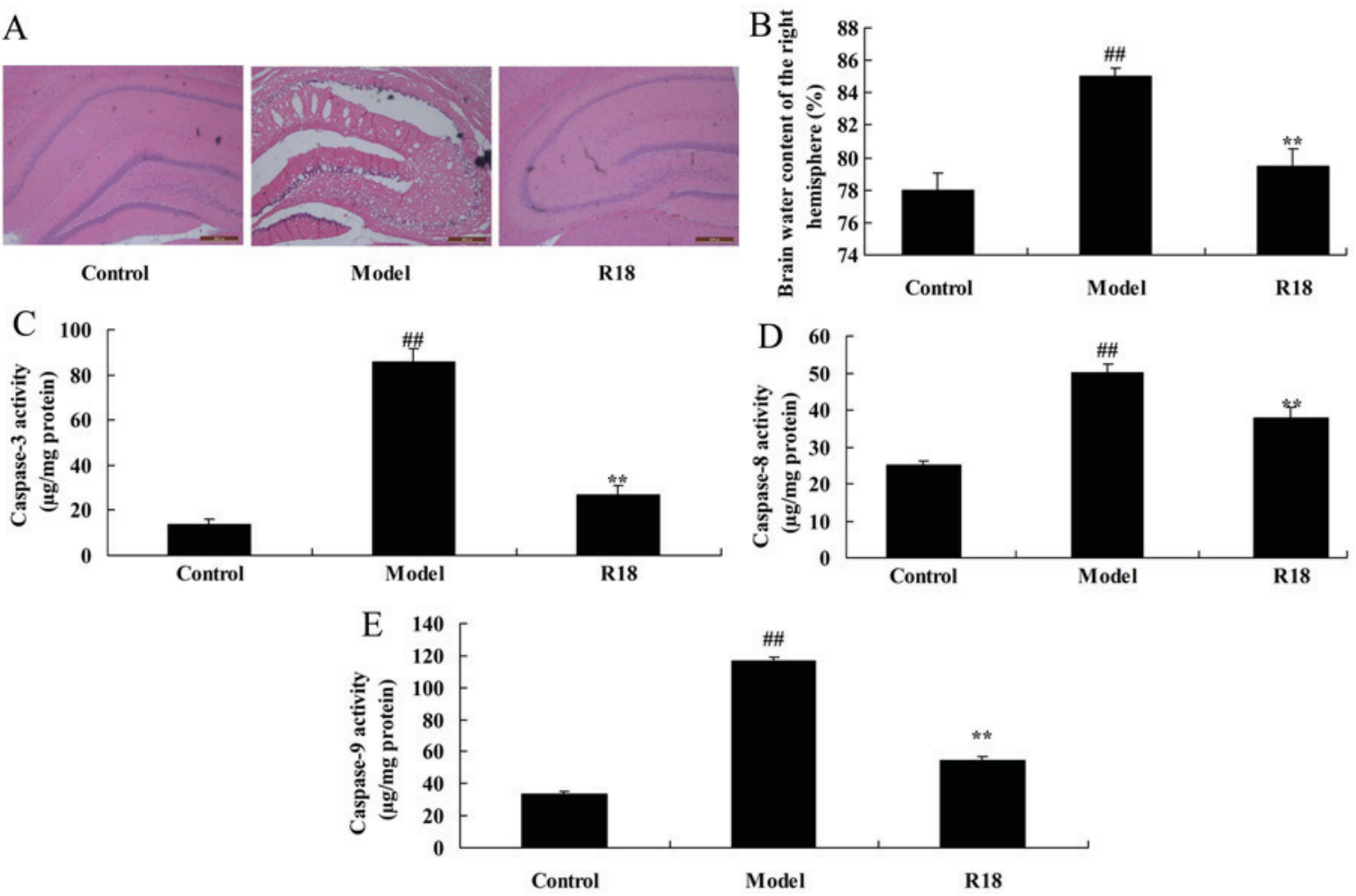

Figure 1. Poly-arginine R18 treatment promotes neurocyte cell growth in TBI rats. (A) Detection of apoptosis by H\&E staining in brain tissue obtained from

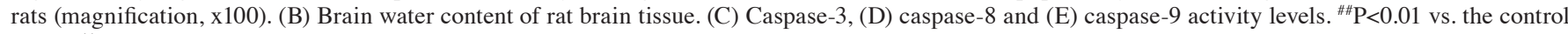
group; ${ }^{* *} \mathrm{P}<0.01 \mathrm{vs}$. the model group. TBI, traumatic brain injury; model, traumatic brain injury rats; R18, poly-arginine $\mathrm{R} 18$ treated rats.
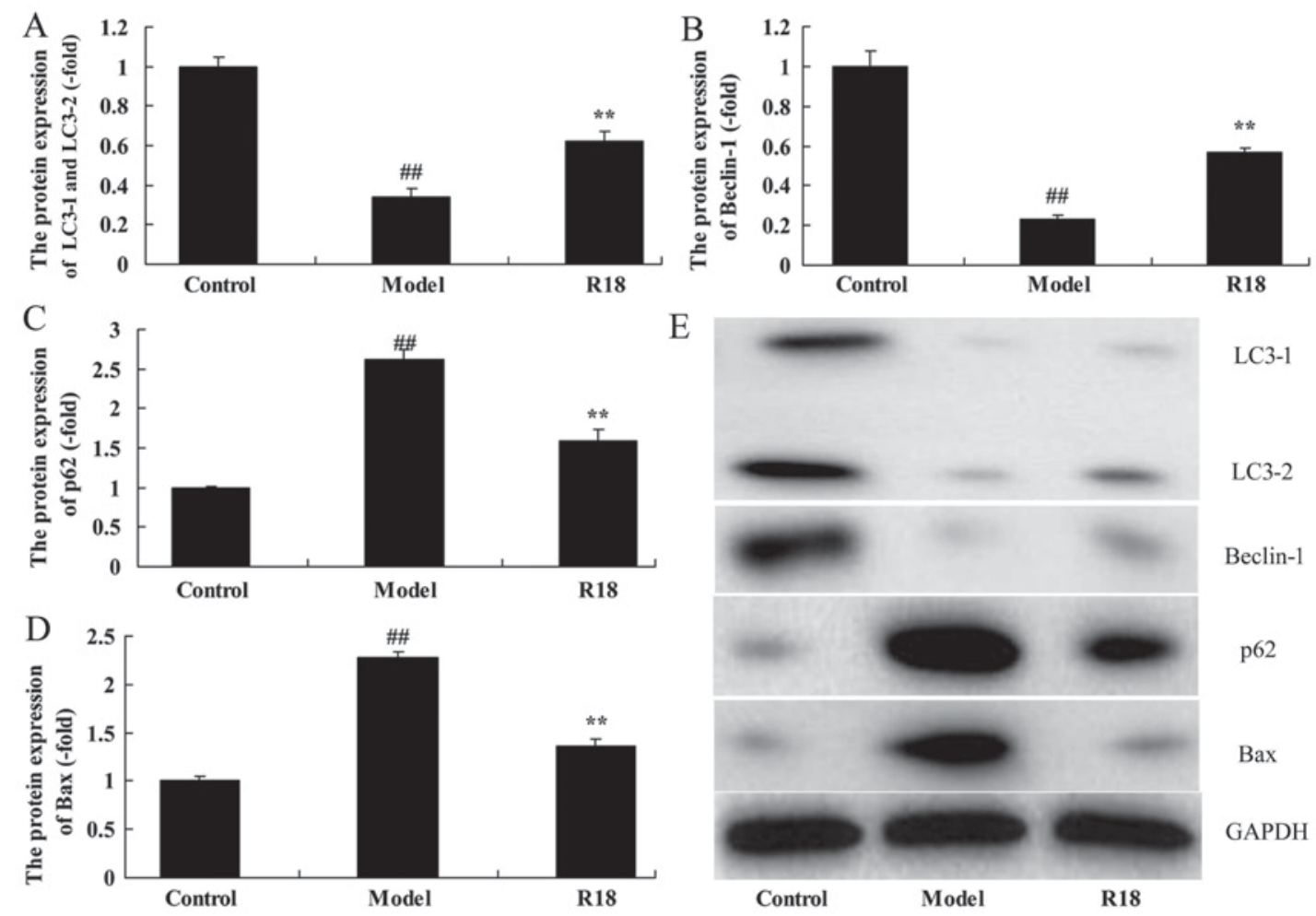

Figure 2. Poly-arginine R18 treatment induces neurocyte cell autophagy in TBI rats. Quantification of (A) LC3-1 and LC3-2, (B) Beclin-1, (C) p62 and (D) Bax protein expression and (E) western blot analysis. ${ }^{\# /} \mathrm{P}<0.01$ vs. the control group; ${ }^{* *} \mathrm{P}<0.01$ vs. the model group. TBI, traumatic brain injury; Bax, Bcl-2 associated $\mathrm{X}$; model, traumatic brain injury rats; R18, poly-arginine $\mathrm{R} 18$ treated rats.

Autophagy is involved in the pathological and physiological process of numerous diseases. However, no consensus has been reached on the effect of autophagy on organ function (16). A previous study has indicated that autophagy may 

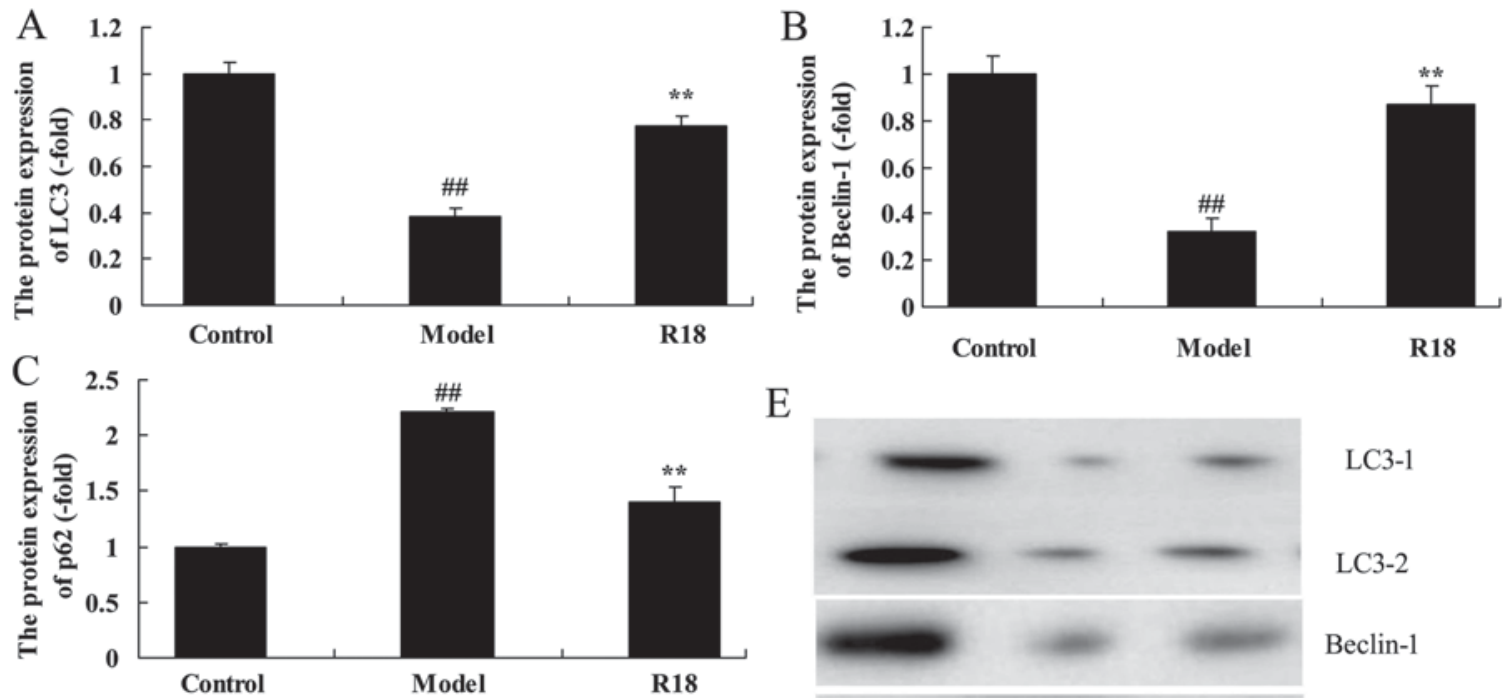

$\mathrm{E}$
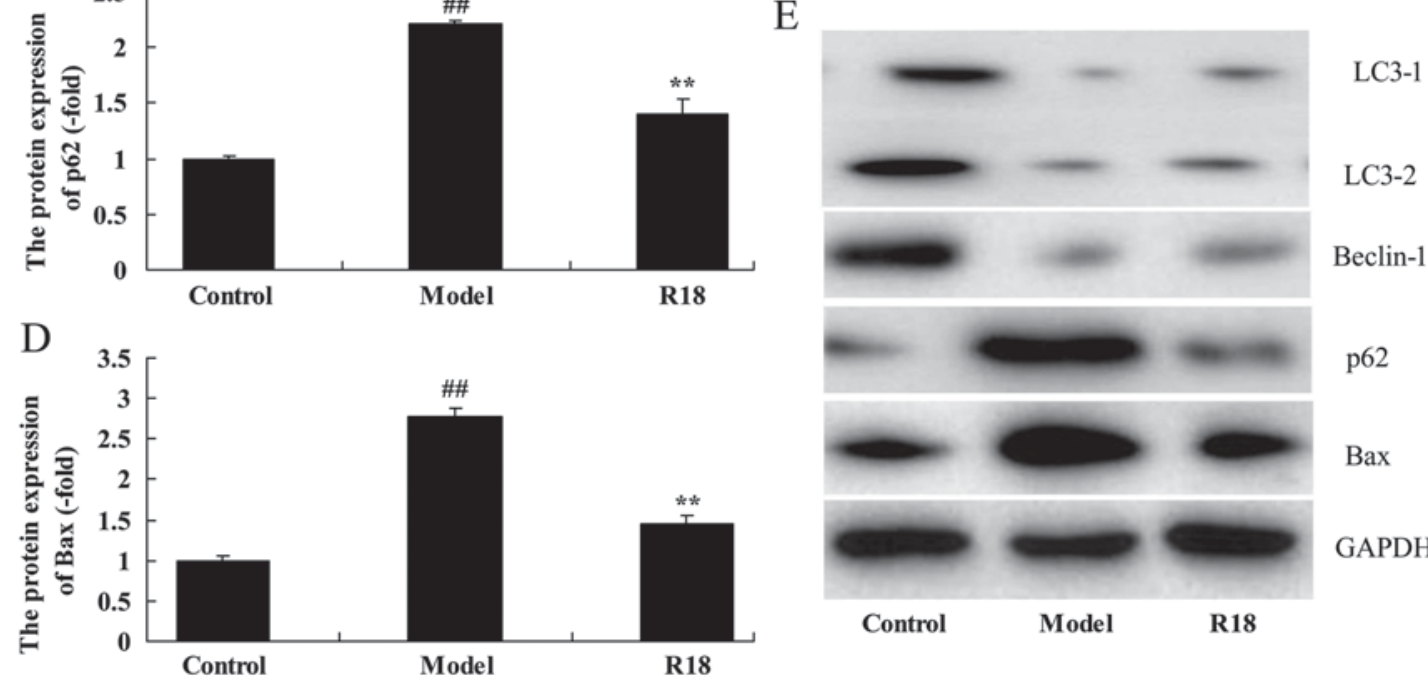

p62

$\operatorname{Bax}$

GAPDH

Figure 3. Poly-arginine R18 treatment induces neurocyte cell autophagy in an in vitro model. Quantification of (A) LC3, (B) Beclin-1, (C) p62 and (D) Bax protein expression and (E) western blot analysis. ${ }^{\# \#} \mathrm{P}<0.01$ vs. the control group; ${ }^{* *} \mathrm{P}<0.01$ vs. the model group. Bax, Bcl-2 associated $\mathrm{X}$; N2A, neuro-2A; model, N2A cells treated with $500 \mathrm{ng} / \mathrm{ml}$ LPS; R18, cells treated with $500 \mathrm{ng} / \mathrm{ml}$ LPS and $0.5 \mu \mathrm{M}$ poly-arginine R18. LPS, lipopolysaccharide.
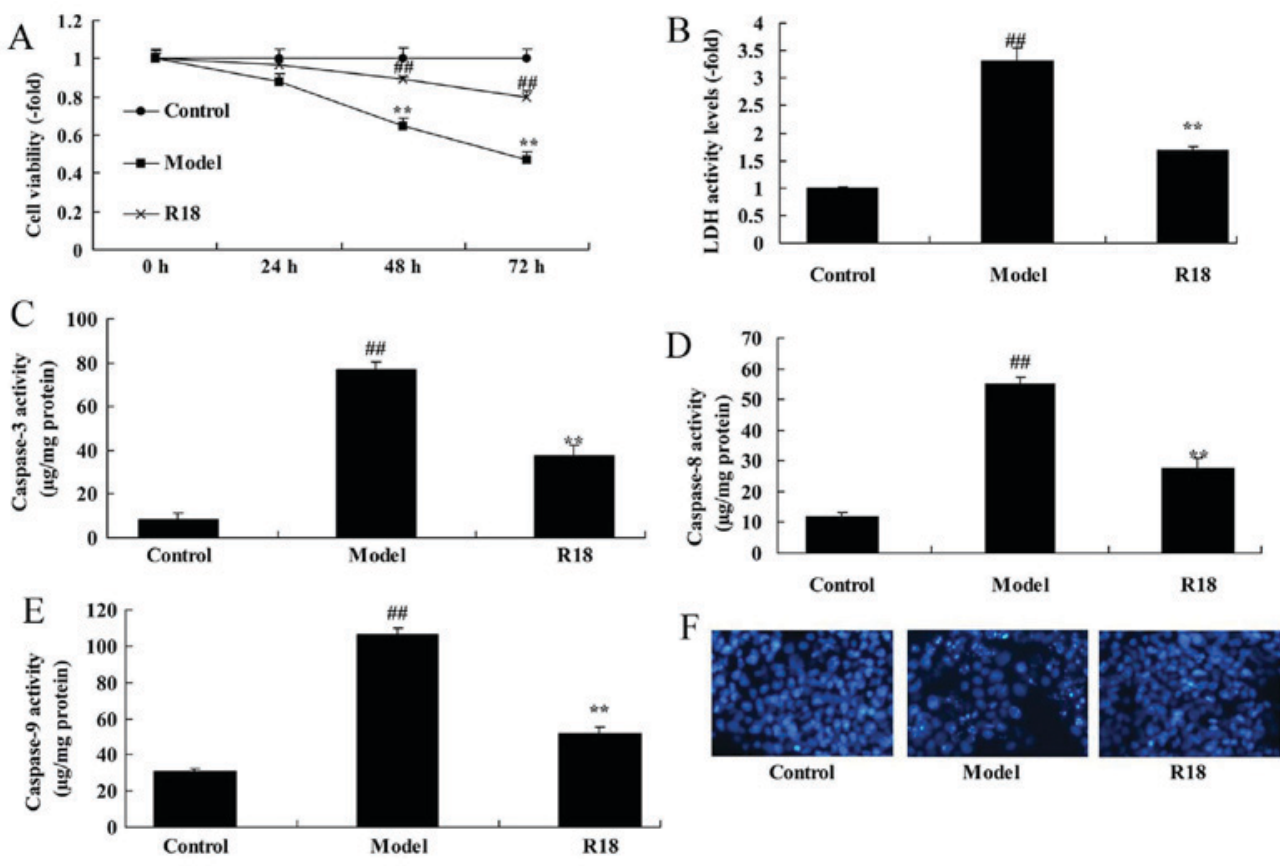

Control

Model

R18

Figure 4. Poly-arginine R18 promotes neurocyte cell proliferation and reduces LDH activity in an in vitro model. (A) Cell proliferation, (B) LDH and (C) caspase-3, (D) caspase-8 and (E) caspase-9 activity was assessed in N2A cells. (F) DAPI staining. ${ }^{\# \#} \mathrm{P}<0.01$ vs. the control group; ${ }^{* * *} \mathrm{P}<0.01$ vs. the model group. LDH, lactate dehydrogenase; N2A, neuro-2A; model, N2A cells treated with $500 \mathrm{ng} / \mathrm{ml}$ LPS; R18, cells treated with $500 \mathrm{ng} / \mathrm{ml} \mathrm{LPS}$ and $0.5 \mu \mathrm{M}$ poly-arginine R18. LPS, lipopolysaccharide.

lead to autophagic cell death or apoptosis, thus aggravating organ dysfunction (17). A previous study demonstrated that autophagy eliminates damaged mitochondria, blocks the apoptotic factor from being released into plasma and exhibits 

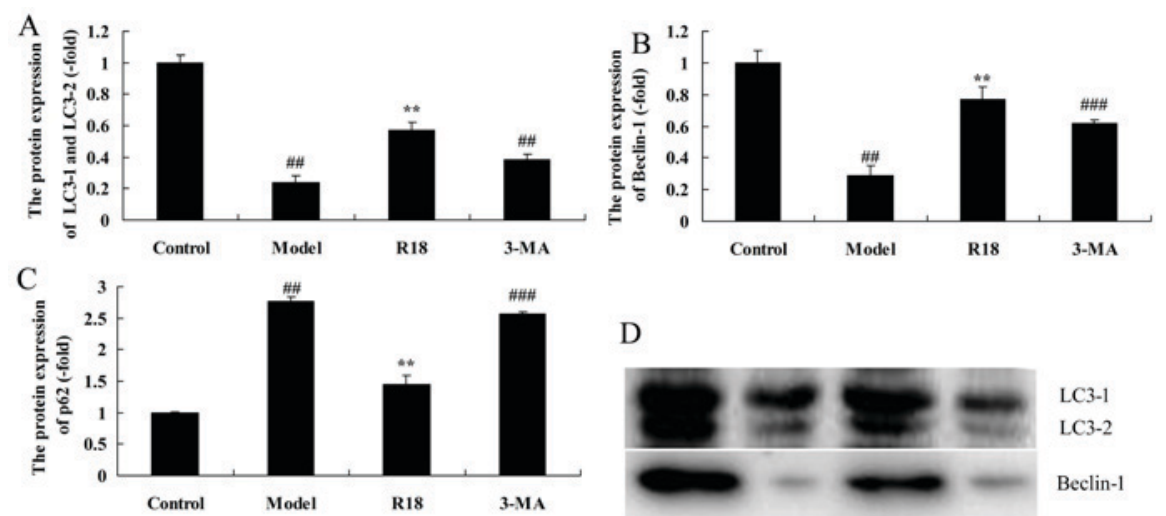

D
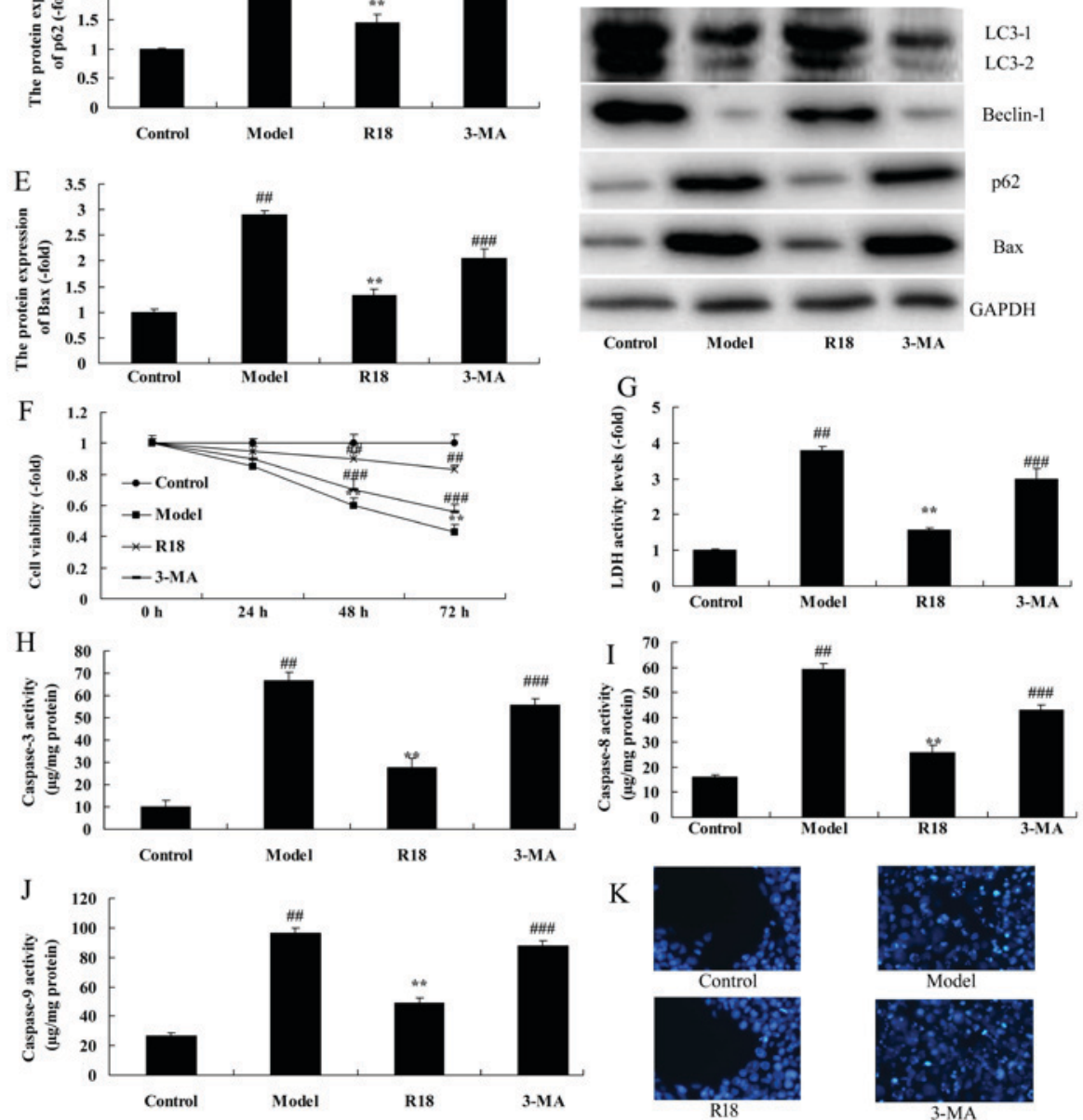

K
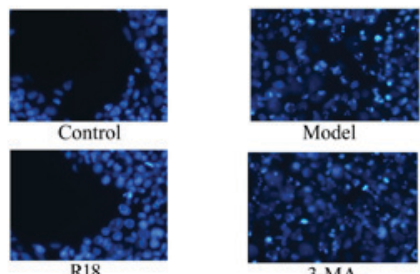

Figure 5. Inhibition of autophagy reduces the effect of poly-arginine R18 on cell growth in an in vitro model. Quantification of (A) LC3, (B) Beclin-1 and (C) p62 protein expression and (D) western blotting. (E) Bax expression was also assessed. (F) Cell proliferation, (G) LDH activity and (H) caspase-3, (I) caspase-8 and (J) caspase-9 activity was assessed in N2A cells. (K) DAPI staining. ${ }^{\# \#} \mathrm{P}<0.01$ vs. the control group; ${ }^{* *} \mathrm{P}<0.01$ vs. the model group; ${ }^{\# \# / "} \mathrm{P}<0.01$ vs. the R18 group. Bax, Bcl-2 associated X; LDH, lactate dehydrogenase; N2A, neruo-2A; model, N2A cells treated with 500 ng/ml LPS; R18, N2A cells treated with $500 \mathrm{ng} / \mathrm{ml}$ LPS and $0.5 \mu \mathrm{M}$ poly-arginine R18; 3-MA, N2A cells treated with $500 \mathrm{ng} / \mathrm{ml} \mathrm{LPS,} 0.5 \mu \mathrm{M}$ poly-arginine R18 and $5 \mu \mathrm{M} 3-\mathrm{MA}$. LPS, lipopolysaccharide.

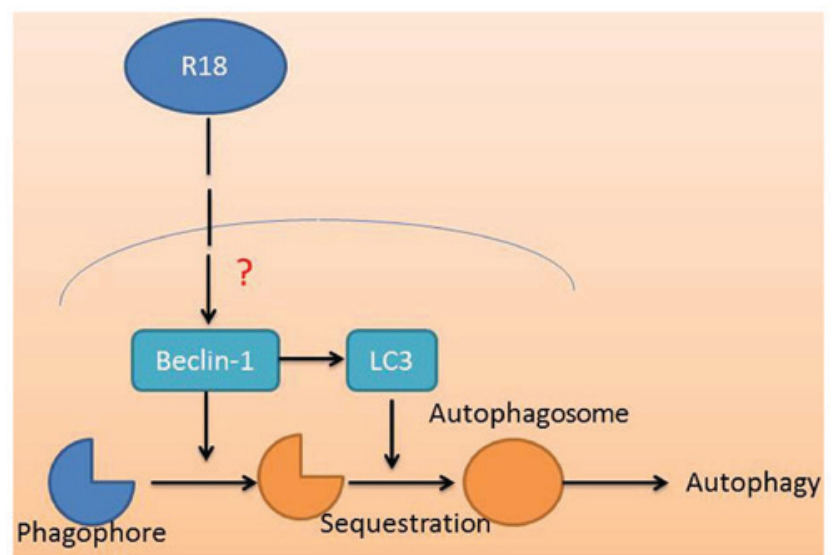

Figure 6. Effect of poly-arginine R18 on neurocyte cell growth in TBI may be through autophagy activation. 
protection (18). The role of autophagy in the protection or aggravation of injury depends on the precise location of autophagy and the stage of autophagy (18). Typically, excessive autophagy following brain injury leads to poor neurological function. In the present study, the inhibition of autophagy was revealed to reduce the effects of poly-arginine R18 on cell proliferation in an in vitro model.

In conclusion, the current study demonstrated that poly-arginine R18 treatment reduces neurocyte cell apoptosis and brain water content, and reduces caspase-3/8/9 activity levels in TBI rats. Therefore, treatment with poly-arginine R18 may induce neurocyte cell autophagy and promote neurocyte cell growth in TBI rats (Fig. 6). These findings may have clinical significance and may therefore justify the further assessment of poly-arginine R18 as a novel class of neuroprotective agent in the regulation of autophagy in TBI.

\section{Acknowledgements}

Not applicable.

\section{Funding}

No funding was received.

\section{Availability of data and materials}

The datasets used and/or analyzed during the current study are available from the corresponding author on reasonable request.

\section{Authors' contributions}

DG designed the experiment; HB, GD, DL, DS and YF performed the experiment. DG and HB analyzed the data; DG prepared the manuscript.

\section{Ethics approval and consent to participate}

All experimental procedures in the current study were approved by the Animal Ethics Committee of the First Affiliated Hospital of Xinjiang Medical University (Xinjiang, China).

\section{Patient consent for publication}

Not applicable.

\section{Competing interests}

The authors declare that they have no competing interests.

\section{References}

1. Yang Z, Lin F, Robertson CS and Wang KK: Dual vulnerability of TDP-43 to calpain and caspase-3 proteolysis after neurotoxic conditions and traumatic brain injury. J Cereb Blood Flow Metab 34: 1444-1452, 2014

2. Okonkwo DO, Shutter LA, Moore C, Temkin NR, Puccio AM, Madden CJ, Andaluz N, Chesnut RM, Bullock MR, Grant GA, et al: Brain oxygen optimization in severe traumatic brain injury Phase-II: A Phase II randomized trial. Crit Care Med 45: 1907-1914, 2017.
3. Grima NA, Rajaratnam SMW, Mansfield D, Sletten TL, Spitz G and Ponsford JL: Efficacy of melatonin for sleep disturbance following traumatic brain injury: A randomised controlled trial. BMC Med 16: 8, 2018.

4. Sun L, Zhao M, Wang Y, Liu A, Lv M, Li Y, Yang X and Wu Z: Neuroprotective effects of miR-27a against traumatic brain injury via suppressing FoxO3a-mediated neuronal autophagy. Biochem Biophys Res Commun 482: 1141-1147, 2017.

5. Zeng XJ, Li P, Ning YL, Zhao Y, Peng Y, Yang N, Zhao ZA, Chen JF and Zhou YG: Impaired autophagic flux is associated with the severity of trauma and the role of $\mathrm{A}_{2 \mathrm{~A}} \mathrm{R}$ in brain cells after traumatic brain injury. Cell Death Dis 9: 252, 2018.

6. Chen X, Wang H, Zhou M, Li X, Fang Z, Gao H, Li Y and Hu W: Valproic acid attenuates traumatic brain injury-induced inflammation in vivo: Involvement of autophagy and the Nrf2/ARE signaling pathway. Front Mol Neurosci 11: 117, 2018.

7. Milani D, Knuckey NW, Anderton RS, Cross JL and Meloni BP: The R18 polyarginine peptide is more effective than the TAT-NR2B9c (NA-1) peptide when administered 60 minutes after permanent middle cerebral artery occlusion in the rat. Stroke Res Treat 2016: 2372710, 2016.

8. Cui C, Cui J, Jin F, Cui Y, Li R, Jiang X, Tian Y, Wang K, Jiang P and Gao J: Induction of the Vitamin D receptor attenuates autophagy dysfunction-mediated cell death following traumatic brain injury. Cell Physiol Biochem 42: 1888-1896, 2017.

9. Milani D, Bakeberg MC, Cross JL, Clark VW, Anderton RS, Blacker DJ, Knuckey NW and Meloni BP: Comparison of neuroprotective efficacy of poly-arginine R18 and R18D (D-enantiomer) peptides following permanent middle cerebral artery occlusion in the Wistar rat and in vitro toxicity studies. PLoS One 13: e0193884, 2018.

10. Blaha RZ, Arnett AB, Kirkwood MW, Taylor HG, Stancin T, Brown TM and Wade SL: Factors influencing attrition in a multisite, randomized, clinical trial following traumatic brain injury in adolescence. J Head Trauma Rehabil 30: E33-E40, 2015.

11. Hammond FM, Alexander DN, Cutler AJ, D'Amico S, Doody RS, Sauve W, Zorowitz RD, Davis CS, Shin P, Ledon F, et al: PRISM II: An open-label study to assess effectiveness of dextromethorphan/quinidine for pseudobulbar affect in patients with dementia, stroke or traumatic brain injury. BMC Neurol 16: 89, 2016.

12. Beca J, McSharry B, Erickson S, Yung M, Schibler A, Slater A, Wilkins B, Singhal A, Williams G, Sherring C, et al: Hypothermia for traumatic brain injury in children-a phase II randomized controlled trial. Crit Care Med 43: 1458-1466, 2015.

13. Renaud MI,Lambregts SA, de Kloet AJ, Catsman-Berrevoets CE, van de Port IG and van Heugten CM: Activities and participation of children and adolescents after mild traumatic brain injury and the effectiveness of an early intervention (Brains Ahead!): Study protocol for a cohort study with a nested randomised controlled trial. Trials 17: 236, 2016

14. Chiu LS, Anderton RS, Cross JL, Clark VW, Edwards AB, Knuckey NW and Meloni BP: Assessment of R18, COG1410, and APP96-110 in excitotoxicity and traumatic brain injury. Transl Neurosci 8: 147-157, 2017

15. Wolf MS, Bayir H, Kochanek PM and Clark RSB: The role of autophagy in acute brain injury: A state of flux? Neurobiol Dis 122: 9-15, 2019.

16. Sun LQ, Gao JL, Cui Y, Zhao MM, Jing XB, Li R, Tian YX, Cui JZ and Wu ZX: Neuronic autophagy contributes to p-connexin 43 degradation in hippocampal astrocytes following traumatic brain injury in rats. Mol Med Rep 11: 4419-4423, 2015.

17. Jin Y, Lin Y, Feng JF, Jia F, Gao G and Jiang JY: Attenuation of cell death in injured cortex after post-traumatic brain injury moderate hypothermia: Possible involvement of autophagy pathway. World Neurosurg 84: 420-430, 2015.

18. Cordaro M, Impellizzeri D, Paterniti I, Bruschetta G, Siracusa R, De Stefano D, Cuzzocrea S and Esposito E: Neuroprotective effects of Co-UltraPEALut on secondary inflammatory process and autophagy involved in traumatic brain injury. J Neurotrauma 33: 132-146, 2016.

This work is licensed under a Creative Commons Attribution-NonCommercial-NoDerivatives 4.0 International (CC BY-NC-ND 4.0) License. 